\title{
Noncausal Multipliers for Nonlinear System Stability
}

\author{
YEDATORE V. VENKATESH
}

\begin{abstract}
Using the Popov approach, new absolute stability conditions in multiplier form are derived for a single-loop system with a time-invariant stable linear element $G$ in the forward path and a nonlinear time-varying gain $k(t) \phi(\cdot)$ in the feedback path. The classes of nonlinearities considered are the monotonic, odd monotonic, and power law. The stability multiplier contains causal and noncausal terms; for absolute stability, the latter give rise to a lower bound (which is believed to be new) on $d k / d t$ and the former, as in earlier investigations, to an upper bound on $d k / d t$. Asymptotic stability conditions for a linear system are realized as a limiting case of the absolute stability conditions derived for the power law nonlinearity.
\end{abstract}

\section{INTRODUCTION}

$\mathbf{T}$ HE FEEDBACK system of Fig. 1 contains a stable time-invariant block $G$ and a nonlinear time-varying feedback gain $k(t) \phi(\cdot)$. The system is governed by the set of equations

$$
\begin{aligned}
d x / d t & =A x-b k(t) \phi(\sigma) \\
\sigma & =c^{\prime} x
\end{aligned}
$$

where $A$ is an $n \times n$ (constant) stable matrix; $b, c, x$ (state) are $n \times 1$ vectors; $\sigma$ the output of the system is a scalar. The time-varying gain $k(t)$ is assumed to be continuous and bounded; for convenience, it is allowed to belong to the infinite sector or $0<\epsilon \leq k(t)<\infty$. The transfer function of the system is $G(s)=c^{\prime}(s I-A)^{-1} b$. $\phi(\sigma)$ is a memoryless continuous nonlinearity satisfying in general the conditions

$$
\phi(0)=0, \quad \sigma \phi(\sigma)>0, \quad \text { for all } \sigma \neq 0 .
$$

This class of functions is denoted by $P$, i.e., $\phi(\sigma) \subset P$. For simplicity in the proofs of the theorems it is assumed that there exist positive constants $h_{1}$ (however small) and $h_{2}$ such that

$$
h_{1} \sigma^{2} \leq \sigma \phi(\sigma)<h_{2} \sigma^{2}
$$

If $\phi(\sigma)$ satisfies additional conditions, then it belongs to other classes. For example,

1) $\phi(\sigma) \subset P_{M}$, the class of monotonically nondecreasing functions

$$
\left[\phi\left(\sigma_{1}\right)-\phi\left(\sigma_{2}\right)\right]\left(\sigma_{1}-\sigma_{2}\right) \geq 0, \text { for all } \sigma_{1} \text { and } \sigma_{2}
$$

Manuscript received June 26, 1969.

The author is with the Department of Electrical Engineering, Indian Institute of Science, Bangalore 12, India.

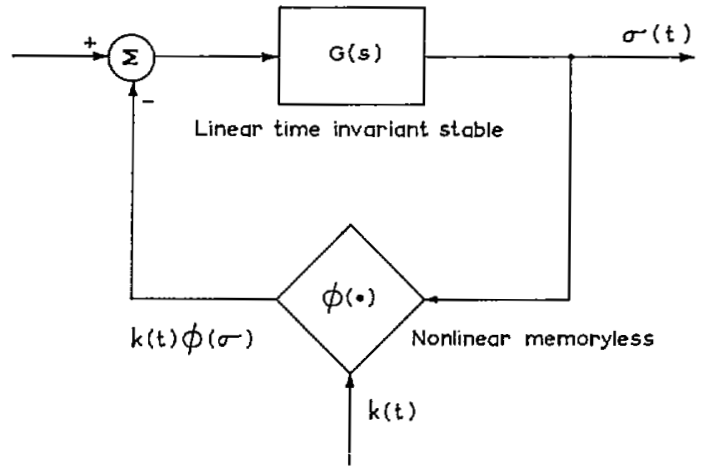

Fig. 1. Nonlinear time-varying feedback system.

2) $\phi(\sigma) \subset P_{M O}$, the class of odd monotonically nondecreasing functions (2) and

$\phi\left(\sigma_{1}\right)\left(\sigma_{1}+\sigma_{2}\right)-\phi\left(\sigma_{2}\right)\left(\sigma_{1}-\sigma_{2}\right) \geq 0$, for all $\sigma_{1}$ and $\sigma_{2}$

3) $\phi(\sigma) \subset P_{P}^{m a}$, the class of power law functions

$$
\begin{aligned}
\phi(\sigma)= & -\phi(-\sigma) \\
\left|\sigma_{1} / \sigma_{2}\right|^{1 / m_{0}} \leq \mid \phi\left(\sigma_{1}\right) / \phi\left(\sigma_{2}\right) ! & \leq \mid \sigma_{1} / \sigma_{2}{ }_{i}^{\prime m_{0}}, \\
& \text { for }{ }^{\prime} \sigma_{1}|\geq| \sigma_{2} \mid \text { and } m_{0} \geq 1 .
\end{aligned}
$$

Or, equivalently, (2) and

$\phi\left(\sigma_{1}\right)\left(c \sigma_{1}+\sigma_{2}\right)+\phi\left(\sigma_{2}\right)\left(c \sigma_{2}-\sigma_{1}\right) \geq 0$, for all $\sigma_{1}$ and $\sigma_{2}$

where

$$
c=\max _{0<\sigma<\infty}\left|\frac{\sigma^{m_{0}}-\sigma}{1+\sigma^{m_{0}+1}}\right| .
$$

As $m_{0} \rightarrow \infty, c \rightarrow 1$ so that $\phi(\sigma) \subset P_{\text {MO }}$ and (4) reduces to (3); as $m_{0} \rightarrow 1, c \rightarrow 0$ giving a linear feedback; $c=$ 0.3536 corresponds to $m_{0}=3$. (See Thathachar et al. [10].)

Assuming that the closed-loop system is asymptotically stable for all constant linear feedback with $k(t) \equiv K C$ $(0, \infty)$, the problem is to find conditions to guarantee the absolute stability of the null solution of (1) when $k(t) \subset$ $(0, \infty)$ and $\phi(\sigma)$ belongs to any one of the preceding classes.

\section{Definitions}

1) A causal (or nonanticipative) system is one whose response to an impulse applied at $t=0$ is nonzero for 
$t \geq 0$ and zero for $t<0$; the complex-frequency function of such a system is said to be causal.

It is to be noted that passive systems (or systems with a positive real impedance function) are necessarily causal.

2) A noncausal (or anticipative) system is one whose response to an impulse applied at $t=0$ is nonzero for $t \leq 0$ and zero for $t>0$; the complex-frequency function of such a system is said to be noncausal.

\section{Historical Credits}

It appears from a survey of literature that Popov's approach [1] has not been applied to the present problem. Using Zames' positive operator theory [2], Cho and Narendra [3], and in a Lyapunov framework, Narendra and Taylor [4] derived absolute stability conditions in terms of positive real (or causal) multipliers and a local bound on $d k / d t$ (which depends upon $\phi(\sigma)$ and the multiplier employed). But they do not consider a noncausal multiplier and hence their results are less general than those presented in Section III.

Major contents of this paper are the following.

1) A new method is presented for establishing the nonnegativeness of the integral

$$
\int_{0}^{T} k(t) \phi(\sigma)[Z \sigma(t)] d t
$$

mith $Z$ the operator representation of the multiplier chosen. This method easily accommodates noncausal operators in contrast with Zames' [2] and Narendra and Taylor's [4] approaches.

2) Theorems 3 and 4 are presented (in Section III), which contain in part a special noncausal multiplier. The resulting additional lower bound on $d k / d t$ appears to be the first of its kind.

The paper is divided into two main parts: the first part introduces the Popov approach and deals with causal multipliers; Theorem 1, containing an RL-RC multiplier, is proved in detail to provide motivation for the second part, which deals with causal and noncausal multipliers. The proofs of the main results (Theorems 3 and 4) do not differ very much from the proof of Theorem 1; therefore, only the necessary changes are indicated.

\section{Solution of the Main \\ Problem-Causal Mulitiplier}

\section{A. Introduction to Popov Approach}

The integral equation representation of the system is

$$
\begin{aligned}
x(t)=\exp (A t) x(0) & \\
& -\int_{0}^{t} \exp [A(t-\tau)] b k(\tau) \phi(\sigma(\tau)) d \tau
\end{aligned}
$$

$\sigma(t)=c^{\prime} \exp (A t) x(0)$

$$
-\int_{0}^{t} c^{\prime} \exp [A(t-\tau)] b k(\tau) \phi(\sigma(\tau)) d \tau .
$$

The absolute stability of the null solution (NS) of (5) implies the absolute stability of the NS of (1). It is obvious that a knowledge of the behavior of $\sigma(t)$ enables one to deduce the behavior of $x(t)$ from (5a); for example, if $\sigma(t)$ is bounded, so is $x(t)$, that is, all the components of $x(t)$ are bounded; if $\sigma(t)$ tends to zero, so do the components of $x(t)$.

The method used by Popov [1] is to obtain an integral inequality of the form

$$
a(|\sigma|)+\int_{0}^{t} b(|\sigma|) d t<d(|\sigma(0)|)
$$

where $a(r), b(r)$, and $d(r)$ are continuous functions zero in the origin, the first two functions being monotonically increasing. From inequality (6), the absolute stability of the XS of (1) can be proved [1].

In order to realize the integral inequality of the desired form (6), a quadratic functional $\rho(T)$ is considered in $\sigma$ for which one seeks, firstly, a lower bound of the form

$$
a(|\sigma|)+\int_{0}^{t} b(|\sigma|) d t+d_{1}(|\sigma(0)|)
$$

(where $d_{1}(|\sigma(0)|)$ is a function of the initial condition $\sigma(0)$ ) and, secondly, an upper bound of the form $d_{2}\left(\mid \sigma(0)_{1}\right)$. The Fourier transformation is used to obtain this upper bound after a choice (following Popov [1]) of a convenient functional $\rho(T)$.

Let $g(t)=c^{\prime} \exp (A t) b$ be the Laplace inverse of $G(s)$, and $f(t)=c^{\prime} \exp (A t) x(0)$. The assumption of a stable matrix $A$ implies the existence of constants $r_{1}, r_{0}>0$, such that

$$
|g(t)| \leq r_{1} \exp \left(-r_{0} t\right), \quad \text { for all } t \geq 0 .
$$

Also, for the same reason, there exists a constant $r_{2}>0$ such that

$$
|f(t)| \leq r_{2} \exp \left(-r_{0} t\right), \quad t \geq 0 .
$$

Consequently, the Fourier transforms $G(j \omega)$ and $F(j \omega)$ of $g(t)$ and $f(t)$, respectively, exist.

\section{Notation:}

$$
\begin{aligned}
\Phi(\sigma) & =\int_{0}^{\sigma} \phi(\sigma) d \sigma>0, \text { for } \sigma \neq 0 \\
\delta_{\sigma} & =\Phi(\sigma) / \phi(\sigma) \sigma \\
\delta_{\max } & =\max _{\sigma} \Phi(\sigma) / \phi(\sigma) \sigma \\
\psi(\sigma, t) & =k(t) \phi(\sigma) .
\end{aligned}
$$

$\delta_{D}(t-T)$ denotes a unit impulse function occurring at $t=T . Z$ is the operator representation of an impedance function $z(s)$. The small Greek letters $\alpha, \beta, \gamma, \mu, \nu, \lambda, \theta$, $\zeta, \xi$, and $\eta$ are constants. The subscript $i$ when applied to different letters need not have the same range of values, for example,

$$
\sum_{i=1}^{m_{1}} \gamma_{i} ; \quad \sum \sum_{i=1}^{m_{1}} \gamma_{i}^{m_{2}}
$$




\section{B. Mathematical Preliminaries}

\section{Lemma 1}

The integral

$$
I_{0}=\int_{0}^{T} \psi(\sigma, t)[\alpha \sigma(t)+\beta(d \sigma / d t)] d t, \quad \alpha>0, \quad \beta \geq 0
$$

is positive (except for a constant term of the form $d_{1}(|\sigma(0)|)$ appearing in $\left.(7)\right)$ if, for some $\epsilon_{0}>0$,

$$
(d k / d t) \delta_{\max } \leq(\alpha / \beta) k(t)-\epsilon_{0}, \text { for all } t .
$$

Proof:

$$
I_{0}=\int_{0}^{T} \alpha k(t) \phi(\sigma) \sigma d t+\int_{0}^{T} \beta k(t) \phi(\sigma)(d \sigma / d t) d t .
$$

Integrate the second term by parts to get

$$
\begin{aligned}
I_{0}=\int_{0}^{T}\left[\alpha k(t)-\beta \delta_{\sigma}(d k / d t)\right] \phi(\sigma) \sigma d t \\
\\
\quad+\beta k(T) \Phi(\sigma(T))-\beta k(0) \Phi(\sigma(0))
\end{aligned}
$$

which is positive (except for the last constant term) if (8) is satisfied.

Q.E.D.

Consider

$$
z_{1}(s)=\sum_{i=1}^{m_{1}} \gamma_{i}\left(s+\nu_{i} \mu_{i}\right) /\left(s+\mu_{i}\right)
$$$$
\gamma_{i}, \mu_{i}>0, \quad \nu_{i} \geq 0, \text { for all } i
$$

Lemma 2

For $\phi(\sigma) \subset P_{M}$, the integral

$$
I_{1}=\int_{0}^{T} \psi(\sigma, t)\left[Z_{1} \sigma(t)\right] d t
$$

is nonnegative (except for a constant term of the form $d_{1}(\sigma(0) \mid)$ in $\left.(7)\right)$ if

and

$$
0 \leq \nu_{i}<1, \text { for all } i
$$

$$
(d k / d t) \delta_{\max } \leq \min _{i} \nu_{i} \mu_{i} k(t)
$$

Proof: Let

$$
\begin{aligned}
z_{1 i}(s) & =\left(s+\nu_{i} \mu_{i}\right) /\left(s+\mu_{i}\right) \\
& =1+\left(\nu_{i}-1\right) \mu_{i} /\left(s+\mu_{i}\right) .
\end{aligned}
$$

Its inverse transform

Let

$$
z_{1 i}(t)=\delta_{D}(t)+\left(\nu_{i}-1\right) \mu_{i} \exp \left(-\mu_{i} t\right) .
$$

$$
\begin{aligned}
I_{1 i}= & \int_{0}^{T} \psi(\sigma, t)\left[Z_{1 i} \sigma(t)\right] d t \\
= & \int_{0}^{T} k(t) \phi(\sigma) \\
& \cdot\left\{\sigma(t)+\left(\nu_{i}-1\right) \mu_{i} \int_{0}^{t} \exp \left[-\mu_{i}(t-\tau)\right] \sigma(\tau) d \tau\right\} d t
\end{aligned}
$$

so that

$$
I_{1}=\sum_{i} \gamma_{i} I_{1 i}
$$

When $\phi(\sigma) \subset P_{M},(2)$ is satisfied. In (11), let

$\sigma_{1}=\sigma$
$\sigma_{1}-\sigma_{2}=\sigma(t)+\left(\nu_{i}-1\right) \mu_{i} \int_{0}^{t} \exp \left[-\mu_{i}(t-\tau)\right] \sigma(\tau) d \tau$ from which

$$
\sigma_{2}=-\mu_{i}\left(\nu_{i}-1\right) \int_{0}^{t} \exp \left[-\mu_{i}(t-\tau)\right] \sigma(\tau) d \tau
$$

and

or

$$
d \sigma_{2} / d t=-\mu_{i} \sigma_{2}+\mu_{i}\left(1-\nu_{i}\right) \sigma(t)
$$

$$
\sigma(t)=\sigma_{1}(t)=\frac{d \sigma_{2} / d t+\mu_{i} \sigma_{2}}{\mu_{i}\left(1-\nu_{i}\right)}
$$

Adding to and subtracting from (11) the expression $\phi\left(\sigma_{2}\right)\left(\sigma_{1}-\sigma_{2}\right)$, one has

$$
\begin{aligned}
I_{1 i}=\int_{0}^{T} k(t)\left[\phi\left(\sigma_{1}\right)-\right. & \left.\phi\left(\sigma_{2}\right)\right]\left(\sigma_{1}-\sigma_{2}\right) d t \\
& +\int_{0}^{T} k(t) \phi\left(\sigma_{2}\right)\left(\sigma_{1}-\sigma_{2}\right) d t
\end{aligned}
$$

The first integral of (13) is nonnegative in virtue of (2); the second integral on using (12) becomes

$$
\begin{aligned}
& \int_{0}^{T} k(t) \phi\left(\sigma_{2}\right)\left(\sigma_{1}-\sigma_{2}\right) d t \\
& \quad=\frac{1}{\left(1-\nu_{i}\right) \mu_{i}} \int_{0}^{T} k(t) \phi\left(\sigma_{2}\right)\left[d \sigma_{2} / d t+\nu_{i} \mu_{i} \sigma_{2}\right] d t
\end{aligned}
$$

When $k(t)$ is a constant, integral (14) (and hence $I_{1}$ ) is nonnegative if $0 \leq \nu_{i}<1$ (for all $i$ ), thereby verifying earlier results [o], [6]. However, when $k(t)$ is time-varying, use Lemma 1 to get (10) for the nonnegativity of $I_{1}$.

Consider

Q.E.D.

$z_{2}(s)=\sum_{i=1}^{m_{2}} \gamma_{i}{ }^{\prime}\left(s+\nu_{i}{ }^{\prime} \mu_{i}^{\prime}\right) /\left(s+\mu_{i}{ }^{\prime}\right)$,

$$
\gamma_{i}{ }^{\prime}, \mu_{i}^{\prime}>0, \quad \nu_{i}^{\prime} \geq 0, \text { for all } i \text {. }
$$

The proof of the following lemma is based on (3) and is similar to the proof of Lemma 2.

\section{Lemma 3}

For $\phi(\sigma) \subset P_{M O}$, the integral

$$
I_{2}=\int_{0}^{T} \psi(\sigma, t)\left[Z_{2} \sigma(t)\right] d t
$$

is nonnegative (except for a constant term of the form $d_{1}(|\sigma(0)|)$ in $\left.(7)\right)$ if

$$
1<\mu_{i}^{\prime}<2, \text { for all } i
$$


and

$$
(d k / d t) \delta_{\max } \leq \min _{i}\left(2-\nu_{i}{ }^{\prime}\right) \mu_{i}{ }^{\prime} k(t) .
$$

Corollary: For $\phi(\sigma) \subset P_{M o}$, the integral

$$
I_{12}=\int_{0}^{T} \psi(\sigma, t)\left[\left(Z_{1}+Z_{2}\right) \sigma(t)\right] d t
$$

is nonnegative (except for a constant term of the form $d_{1}(|\sigma(0)|)$ in (7)) if (10) and (16) are simultaneously satisfied.

The following two lemmas are extensions of the results found in Popov [1]; the proofs are omitted here.

\section{Lemma 4}

If $|f(t)| \leq r_{2} \exp \left(-r_{0} t\right)$, with $r_{2}, r_{0}>0$, then there exists a constant $R_{1}$ independent of $T$ such that

$$
\begin{array}{r}
\left|\int_{0}^{T}\left\{\alpha f(t)+\beta(d f / d t)+\left(Z_{1}+Z_{2}\right) f(t)\right\} \psi(\sigma, t) d t\right| \\
\leq R_{1} \sup _{0 \leq t \leq T}|\sigma(t)| .
\end{array}
$$

\section{Lemma 5 (Popov-Barbalat)}

Let $y(t)$ map $[0, \infty)$ into the real line, and be differentiable. If $y$ and $d y / d t$ are bounded on $[0, x), J(y(t))=\mathbf{0}$, for $y(t)=0$ continuous, and $J(y(t))>0$, for $y(t) \neq 0$, $0<\epsilon_{0} \leq \epsilon(t)$, for all $t$, and

$$
\int_{0}^{\infty} \epsilon(t) J(y(t)) d t<\infty
$$

then

$$
\lim _{t \rightarrow \infty} y(t)=0 .
$$

\section{Absolute Stability}

Based on the preceding preliminaries, the absolute stability conditions for (5) are derived assuming that $\phi(\sigma) \subset P_{\text {uo. }}$. The stability multiplier, being then an RCRL impedance, contains as special cases the multipliers for $\phi(\sigma) \subset P$ and $\phi(\sigma) \subset P_{M}$. Therefore, when $\phi(\sigma) \subset P$ or $P_{M}$, Theorem 1 holds after casting out the inadmissible terms from the multiplier. The functions $z_{1}(s)$ and $z_{2}(s)$ are as defined in (9) and (15), respectively.

\section{Theorem 1}

The system governed by (5) is absolutely stable for $\phi(\sigma) \subset P_{\text {MO }}$ if there exist constant $\alpha, \beta>0 ; \gamma_{i}, \gamma_{i}{ }^{\prime}, \mu_{i}, \mu_{i}{ }^{\prime} \geq 0$; $0 \leq \nu_{i}<1,1<\nu_{i}^{\prime} \leq 2$, for all $i$; and a multiplier

such that

$$
z(s)=\alpha+\beta s+z_{1}(s)+z_{2}(s)
$$

a) $\operatorname{Re} Z(j \omega) G(j \omega) \geq 0$, for all real $\omega$, and, for some $\epsilon_{0}>0$, as small as desired,

b) $(d k / d t) \delta_{\mathrm{max}} \leq \min _{i}\left(\alpha / \beta, \nu_{i} \mu_{i},\left(2-\nu_{i}{ }^{\prime}\right) \mu_{i}{ }^{\prime}\right) k(t)-\epsilon_{0}$.
Proof: Let

$$
\begin{array}{rlrl}
\psi_{T} & =\psi(\sigma, t), & & 0 \leq t \leq T \\
& =0, & & t>T \\
\sigma_{T} & =\sigma(t), & & 0 \leq t \leq T \\
& =0, & & t>T \\
f_{T} & =f(t), & & 0 \leq t \leq T \\
& =\int_{0}^{T} g(t-\tau) \psi(\sigma(\tau), \tau) d \tau, & t>T .
\end{array}
$$

Define

$$
\begin{array}{r}
\rho_{1}(T)=\int_{0}^{T}\{\alpha[\sigma(t)-f(t)]+\beta(d / d t)[\sigma(t)-f(t)]\} \psi(\sigma, t) d t \\
\quad+\int_{0}^{T} \psi(\sigma, t)\left[\left(Z_{1}+Z_{2}\right)(\sigma(t)-f(t))\right] d t \quad(19)
\end{array}
$$

which can be written as

$$
\begin{gathered}
\rho_{1}(T)=\int_{0}^{\infty}\left\{\alpha\left(\sigma_{T}-f_{T}\right)+\beta(d / d t)\left(\sigma_{T}-f_{T}\right)\right\} \psi_{T} d t \\
+\int_{0}^{\infty} \psi_{T}\left[\left(Z_{1}+Z_{2}\right)\left(\sigma_{T}-f_{T}\right)\right] d t
\end{gathered}
$$

Let the Fourier transforms of $\sigma_{T}, \psi_{T}$ be, respectively, $\Sigma_{T}, \Psi_{T}$. By virtue of the truncation and the assumption on $G$, Parseval's theorem is applicable to (20), and on application gives

$$
\rho_{\perp}(T)=-\frac{1}{2 \pi} \int_{-\infty}^{\infty} \operatorname{Re} z(j \omega) G(j \omega) \mid \Psi_{T} i^{2} d \omega .
$$

Since $\left|\Psi_{T}\right|^{2}$ is real, $\rho_{1}(T) \leq 0$ according to hypothesis a) of Theorem 1, implying thereby that

$$
\begin{aligned}
& \int_{0}^{T}[\alpha \sigma(t)+\beta(d \sigma / d t)] \psi(\sigma, l) d t \\
& \quad+\int_{0}^{T} \psi(\sigma, t)\left[\left(Z_{1}+Z_{2}\right) \sigma(t)\right] d t \\
& \quad \leq \int_{0}^{T}\left\{[\alpha f+\beta(d f / d t)]+\left[\left(Z_{1}+Z_{2}\right) f(t)\right]\right\} d t
\end{aligned}
$$

Consider the left-hand side of the inequality (22): from Lemma 1, satisfaction of (8) guarantees the positiveness of the first integral. Let

$$
\alpha k(t)-\beta(d k / d t) \delta_{\max }=\epsilon_{1}(t) \geq \epsilon_{0}>0, \text { for all } t .
$$

The second integral of (22) is nonnegative (from Corollary of Lemma 3) if (10) and (16) are simultaneously satisfied. As for the right-hand side of (22), Lemma 4 gives its upper bound in the inequality (17). 
Consequently, (22) becomes

$$
\begin{gathered}
\int_{0}^{T} \epsilon_{1}(t) \phi(\sigma) \sigma d t+\beta k(T) \Phi(\sigma(T)) \\
\quad+\int_{0}^{T} \psi(\sigma, t)\left[\left(Z_{1}+Z_{2}\right) \sigma(t)\right] d t \\
\leq \beta k(0) \Phi(\sigma(0))+R_{1} \sup _{0 \leq t \leq T}|\sigma(t)| .
\end{gathered}
$$

By assumption, there exists a positive constant $h_{1}$ such that $\phi(\sigma) \sigma \geq h_{1} \sigma^{2}$, from which $\Phi(\sigma) \geq h_{1} \sigma^{2} / 2$. Let $\beta_{1}=$ $\beta h_{1} k(T) / 2 ; R_{0}=\beta k(0) \Phi(\sigma(0))$. Then the crucial inequality (23) takes the form

$$
\begin{aligned}
\int_{0}^{T} \epsilon_{1}(t) \phi(\sigma) \sigma d t+\beta_{1} \sigma^{2}(T) & +\int_{0}^{T} \psi(\sigma, t)\left[\left(Z_{1}+Z_{2}\right) \sigma(t)\right] d t \\
& \leq R_{0}+R_{1} \sup _{0 \leq t \leq T} \sigma(t) \mid
\end{aligned}
$$

where constants $R_{0}, R_{1}$ are independent of $T$. Boundedness of $\sigma(t)$ and asymptotic stability follow from (24) as in Popov's proof $[1]$.

Corollary $1: \phi(\sigma) \subset P, z_{1}(s)$ and $z_{2}(s)$ are inadmissible. Hypothesis b) reads

$$
(d k / d t) \delta_{\max } \leq(\alpha / \beta) k(t)-\epsilon_{0} .
$$

Tote that, for a general $\phi(\sigma) \subset P, 0<\delta_{\max } \leq \infty$.

Corollary 2: $\phi(\sigma) \subset P_{M}, z_{2}(s)$ is inadmissible. Hypothesis b) accordingly reads

$$
(d k / d t) \delta_{\max }<\min _{i}\left(\alpha / \beta, \nu_{i} \mu_{i}\right) k(t)-\epsilon_{0} .
$$

Note that, for $\phi(\sigma) \subset P_{M}, 0<\delta_{\max } \leq 1$.

\section{Power Law Nonlinearity (Class $P_{P}{ }^{m 0}$ )}

Preliminaries: In view of the inequality (2) characterizing $\phi(\sigma) \subset P_{P}^{m_{0}}$, Lemma 2 holds here, but Lemma 3 needs modification, as it is based on (3) instead of (4). The proof is similar to that of Lemma 3 .

\section{Lemma 6}

For $\phi(\sigma) \subset P_{P}^{m_{0}}$, the integral

$$
I_{3}=\int_{0}^{T} \psi(\sigma, t)\left[Z_{2} \sigma(t)\right] d t
$$

is nonnegative (except for a constant term of the form $d_{1}(|\sigma(0)|)$ in $\left.(7)\right)$ if

$$
\begin{aligned}
1<\nu_{i}{ }^{\prime} & \leq\left(1+1 / c^{2}\right), \quad \text { for all } i \\
(d k / d t) \delta_{\max } & \leq \min _{i}\left(1+c^{2}-\nu_{i}{ }^{\prime} c^{2}\right) \mu_{i}{ }^{\prime} k(t) .
\end{aligned}
$$

Corollary: When $\phi(\sigma) \subset P_{P}^{m o}$, the integral

$$
I_{13}=\int_{0}^{T} \psi(\sigma, t)\left[\left(Z_{1}+Z_{2}\right) \sigma(t)\right] d t
$$

is nonnegative (except for a constant term of the form $d_{1}(|\sigma(0)|)$ in $\left.(7)\right)$ if (10) and (25) are simultaneously obeyed.
Based on the preceding results, the following theorem generalizes Theorem 1. Its proof, being analogous to the proof of Theorem 1, is omitted.

\section{Theorem 2}

The system governed by (5) is absolutely stable for $\phi(\sigma) \subset P_{P}{ }^{m_{0}}$, if there exist constants $\alpha, \beta>0 ; \gamma_{i}, \gamma_{i}{ }^{\prime}, \mu_{i}$, $\mu_{i}{ }^{\prime} \geq 0 ; 0 \leq \nu_{i}<1,1<\nu_{i}{ }^{\prime} \leq\left(1+1 / c^{2}\right)$, for all $i$, and a multiplier $z(s)=\alpha+\beta s+z_{1}(s)+z_{2}(s)$ such that

a) $\operatorname{Re} z(j \omega) G(j \omega) \geq 0$, for all real $\omega$, and, for some $\epsilon_{0}>0$, as small as desired,

b) $(d k / d t) \delta_{\max } \leq \min _{i}\left(\alpha / \beta, \nu_{i} \mu_{i},\left(1+c^{2}-\nu_{i}{ }^{\prime} c^{2}\right) \mu_{i}{ }^{\prime}\right)$

$$
\cdot k(t)-\epsilon_{0}
$$

Corollary 1: If $\phi(\sigma) \subset P_{\text {uro, }}$, replace $c$ by 1 to get Theorem 1.

Corollary 2: If $\phi(\sigma)=\sigma, c=0$, and $\delta_{\max }=1 / 2$, Theorem 2 then gives conditions for the asymptotic stability of the linear system. In this case, $\nu_{i}^{\prime}>1$, for all $i$, and hypothesis b) reads

$$
d k / d t \leq 2 \min _{i}\left(\alpha / \beta, \nu_{i} \mu_{i}, \mu_{i}^{\prime}\right) k(t)-\epsilon_{0} .
$$

Remark: It is to be observed that the multiplier of Corollary 2 is not a general positive real function (which is in fact expected in view of Gruber and Willems' result [8]). The asymptotic stability conditions for the linear system obtained by a limit process are consequently not the best possible.

\section{Solution of the Main Problem-Causal and Noncaudal Multipliers}

\section{A. Preliminaries}

1) Consider the impulse response function

$$
\begin{array}{ll}
\sum_{i=1}^{m_{3}}-\xi_{i} \exp \left(\zeta_{i} t\right), & t \leq 0, \quad \xi_{i}, \zeta_{i}>0, \quad \text { for all } i \\
0, & t>0
\end{array}
$$

which describes a noncausal system. The Fourier transform exists and is equal to $\sum_{i}-\xi_{i} /\left(\zeta_{i}-j \omega\right)$.

2) Let $\eta_{i} \geq 0$, for all $i$,

$$
\begin{aligned}
\lambda_{i} & =\zeta_{i}-\xi_{i}, \quad \theta_{i}=\lambda_{i} / \zeta_{i} \\
z_{1}{ }^{a}(s) & =\sum_{i} \eta_{i}\left(\zeta_{i} \theta_{i}-s\right) /\left(\zeta_{i}-s\right) \\
& =\sum_{i} \eta_{i}\left[1-\xi_{i} /\left(\zeta_{i}-s\right)\right] .
\end{aligned}
$$

Define

$$
\begin{aligned}
I_{1}{ }^{a} & =\int_{0}^{T} k(t) \phi(\sigma)\left[Z_{1}{ }^{a} \sigma(t)\right] d t \\
& =\int_{0}^{T} k(t) \phi(\sigma)\left(\int_{-\infty}^{0} z_{1}^{a}(\tau) \sigma(t-\tau) d \tau\right) d t \\
& =\int_{0}^{T} k(t) \phi(\sigma)\left(\int_{t}^{\infty} z_{1}{ }^{a}(t-\tau) \sigma(\tau) d \tau\right) d t
\end{aligned}
$$


Let

$$
\begin{aligned}
I_{1 i}{ }^{a}=\int_{0}^{T} k(t) & \phi(\sigma) \\
& (\sigma(t) \\
& \left.-\xi_{i} \int_{t}^{\infty} \exp \left[\zeta_{i}(t-\tau)\right] \sigma(\tau) d \tau\right) d t
\end{aligned}
$$

so that $I_{1}{ }^{a}=\sum_{i} \eta_{i} I_{1 i}{ }^{a}$.

When $\phi(\sigma) \subset P_{M},(2)$ is satisfied. In (28), let $\sigma_{1}=\sigma(t)$, and

$$
\sigma_{1}-\sigma_{2}=\sigma(t)-\xi_{i} \int_{t}^{\infty} \exp \left[\zeta_{i}(t-\tau)\right] \sigma(\tau) d \tau
$$

from which

$$
\sigma_{2}=\xi_{i} \int_{t}^{\infty} \exp \left[\zeta_{i}(t-\tau)\right] \sigma(\tau) d \tau
$$

and

$$
d \sigma_{2} / d t=\xi_{i} \sigma_{2}-\xi_{i} \sigma(t)
$$

or

$$
\sigma(l)=\sigma_{1}=\left(\zeta_{i} \sigma_{2}-d \sigma_{2} / d t\right) / \xi_{i} .
$$

Add to and subtract from (28) the expression $\phi\left(\sigma_{2}\right)\left(\sigma_{1}-\sigma_{2}\right)$ to get

$$
\begin{aligned}
I_{1 i}{ }^{a}=\int_{0}^{T} k(t)\left[\phi\left(\sigma_{1}\right)\right. & \left.-\phi\left(\sigma_{2}\right)\right]\left(\sigma_{1}-\sigma_{2}\right) d t \\
& +\int_{0}^{T} k(t) \phi\left(\sigma_{2}\right)\left(\sigma_{1}-\sigma_{2}\right) d t .
\end{aligned}
$$

If $\phi(\sigma) \subset P_{M}$, the first integral of (31) is nonnegative in virtue of (2). The second integral of (31), on using (30) and integrating by parts, gives

$$
\begin{aligned}
\int_{0}^{T} k(t) \phi\left(\sigma_{2}\right)\left(\sigma_{1}-\sigma_{2}\right) d t \\
=-\left[k(T) \Phi\left(\sigma_{2}(T)\right) / \xi_{i}\right]+k(0) \Phi\left(\sigma_{2}(0)\right) / \xi_{i} \\
\quad+\frac{1}{\xi_{i}} \int_{0}^{T}\left[(d k / d t) \delta_{\sigma_{2}}+\zeta_{i} \theta_{i} k(t)\right] \phi\left(\sigma_{2}\right) \sigma_{2} d t .
\end{aligned}
$$

Since $0<\delta_{\max } \leq 1$ for $\phi(\sigma) \subset P_{L}$ and $k(t) \subset(0, \infty)$, the right-hand side integral of (32) is nonnegative if

$$
(d k / d t) \delta_{\mathrm{max}} \geq-\zeta_{i} \theta_{i} k(t), \text { for all } t .
$$

Consequently, if $\phi(\sigma) \subset P_{M}$ and (33) is satisfied, $I_{1 i^{a}}$ is nonnegative except for the term $-k(T) \Phi\left(\sigma_{2}(T)\right) / \xi_{i}$ in (32). This in turn implies that the inequality

$$
(d k / d t) \delta_{\max } \geq-\min _{i} \zeta_{i} \theta_{i} k(t), \text { for all } t
$$

guarantees the nonnegativity of $I_{1}{ }^{a}$ given in (27), save for the term

$$
\sum_{i}-\eta_{i} k(T) \Phi\left(\sigma_{2}(T)\right) / \xi_{i} .
$$

3) It is now intended to make $I_{1}{ }^{a}$ nonnegative by adding a positive term to (35). To this end, add $\alpha+\beta s$ (with $\alpha, \beta>0$ ) to $z_{1}{ }^{a}(s)$. Let

$$
z_{01}{ }^{a}(s)=\alpha+\beta s+z_{1}^{a}(s)
$$

and define

$$
\begin{aligned}
I_{01}{ }^{a}= & \int_{0}^{T} k(t) \phi(\sigma)\left[Z_{01}{ }^{a} \sigma(t)\right] d t \\
= & \int_{0}^{T}\left[\alpha k(t)-\beta(d k / d t) \delta_{\sigma}\right] \phi(\sigma) \sigma d t \\
& +\beta k(T) \Phi(\sigma(T))-\beta k(0) \Phi(\sigma(0))+I_{1}{ }^{a} .
\end{aligned}
$$

Let it be assumed that (for some $\epsilon_{0}>0$ )

$$
-\min _{i} \zeta_{i} \theta_{i} k(t) \leq(d k / d t) \delta_{\mathrm{Ia} a \mathrm{x}} \leq(\alpha / \beta) k(t)-\epsilon_{0} .
$$

Then, except for a constant term of the form $d_{1}(|\sigma(0)|)$ in (7), (36) is positive if

$$
\beta k(T) \Phi(\sigma(T))-\sum_{i} \eta_{i} k(T) \Phi\left(\sigma_{2}(T)\right) / \xi_{i}>0
$$

or, adding and subtracting $\sum_{2} \eta_{i} k(T) \Phi(\sigma(T)) / \xi_{i}$,

$$
\begin{aligned}
{\left[\beta-\sum_{i}\left(\eta_{i} / \xi_{i}\right)\right] k } & (T) \Phi(\sigma(T)) \\
& +\sum_{i} \eta_{i} \frac{k(T)}{\xi_{i}} \int_{\sigma 3(T)}^{o(T)} \phi(\sigma) d \sigma>0 .
\end{aligned}
$$

The first term of (38) is positive if $\beta>\sum_{i} \eta_{i} / \xi_{i}$. As regards the second term of (38), let $T$ be so chosen that $\sigma(t)$ attains its extremum value $\sigma_{\text {ext }}$ at $t=T$ (i.e., either its positive maximum $\sigma_{\max }{ }^{+}$or its negative maximum $\left.\sigma_{\max }{ }^{-}\right)$. Recalling that

$$
\sigma_{2}(T)=\xi_{i} \int_{T}^{\infty} \exp \left[\zeta_{i}(T-\tau)\right] \sigma(\tau) d \tau
$$

one has

$$
\begin{aligned}
\sigma_{2}(T) & \geq \xi_{i}\left(\sigma_{\max }{ }^{-}\right) \int_{t}^{\infty} \exp \left[\zeta_{i}(T-\tau)\right] d \tau \\
& =\xi_{i} \sigma_{\max }-/ \zeta_{i} \\
\sigma_{2}(T) ! & \leq \xi_{i}\left(\sigma_{\max }{ }^{+}\right) \int_{t}^{\infty} \exp \left[\zeta_{i}(T-\tau)\right] d \tau \\
& =\xi_{i} \sigma_{\max }+/ \zeta_{i} .
\end{aligned}
$$

It is to be noted that $\sigma_{2}(T) \geq \eta \sigma_{\max }{ }^{-}$implies $\sigma_{2}(T)>$ $\sigma_{\text {tana }}-$ if $\eta<1$, and $\left|\sigma_{2}(T)\right| \leq \eta \sigma_{\text {max }}+$ implies $\left|\sigma_{2}(T)\right|<$ $\sigma_{\max }+$ if $\eta<1$. Then, for $0<\theta_{i}<1$ or $\xi_{i}>\xi_{i}$, one concludes from (39) and (40) that $\sigma_{2}(T)>\sigma_{\max }-$ when $\sigma_{2}(T)$ is negative, and $\sigma_{2}(T)<\sigma_{\max }{ }^{+}$when $\sigma_{2}(T)$ is positive. Consequently, the integral

$$
\int_{\sigma_{2}(T)}^{\sigma_{\mathrm{ext}}} \phi(\sigma) d \sigma
$$

is nonnegative by virtue of the fact that $\phi(\sigma) \subset P$. The following is a summary of the preceding findings.

\section{Result 1}

The integral (36) (with $\alpha, \beta>0$, and $T$ so chosen that $\sigma$ attains its extremum value at $t=T$ ) is positive (except for a constant term of the form $d_{1}(|\sigma(0)|)$ in (7)) if 
(for some $\epsilon_{0}>0$ )

$$
\begin{gathered}
-\min _{i} \zeta_{i} \theta_{i} k(t) \leq(d k / d t) \delta_{\max } \leq(\alpha / \beta) k(t)-\epsilon_{0} \\
\beta>\sum_{i} \eta_{i} / \zeta_{i}\left(1-\theta_{i}\right), \quad 0<\theta_{i}<1, \text { for all } i .
\end{gathered}
$$

4) Consider the noncausal impulse response function

$$
\begin{array}{ll}
\sum_{i=1}^{m_{4}} \xi_{i}{ }^{\prime} \exp \left(\zeta_{i}{ }^{\prime} t\right), & t \leq 0, \quad \xi_{i}{ }^{\prime}, \xi_{i}{ }^{\prime}>0, \quad \text { for all } i \\
0, & t>0 .
\end{array}
$$

The Fourier transform exists and is given by

$$
\sum_{i} \xi_{i}{ }^{\prime} /\left(\zeta_{i}^{\prime}-j \omega\right)
$$

Let $\eta_{i}{ }^{\prime} \geq 0$, for all $i ; \lambda_{i}{ }^{\prime}=\zeta_{i}{ }^{\prime}+\xi_{i}{ }^{\prime}, \theta_{i}{ }^{\prime}=\lambda_{i}{ }^{\prime} \zeta_{i}{ }^{\prime} ;$ and

$$
\begin{aligned}
z_{2}{ }^{a}(s) & =\sum_{i} \eta_{i}{ }^{\prime}\left(\theta_{i}{ }^{\prime} \zeta_{i}{ }^{\prime}-s\right) /\left(\zeta_{i}^{\prime}-s\right) \\
& =\sum_{i} \eta_{i}{ }^{\prime}\left[1+\xi_{i}{ }^{\prime} /\left(\zeta_{i}^{\prime}-s\right)\right] .
\end{aligned}
$$

The proof of the following result is similar to that of Result 1 and is hence omitted.

\section{Result 2}

When $\phi(\sigma) \subset P_{x o}$, the integral (with $\alpha, \beta>0$, and $T$ so chosen that $\sigma$ attains its maximum value $\sigma_{\max }$ at $t=T$ )

$$
I_{02}{ }^{a}=\int_{0}^{T} k(t) \phi(\sigma)\left[\alpha \sigma+\beta(d \sigma / d t)+Z_{2}^{a} \sigma(t)\right] d t
$$

is positive (except for a constant term of the form $d_{1}(|\sigma(0)|)$ in $\left.(7)\right)$ if (for some $\epsilon_{0}>0$ )

$$
\begin{gathered}
-\min _{i}\left(2-\theta_{i}\right) \zeta_{i}{ }^{\prime} k(t) \leq(d k / d l) \delta_{\max } \leq \alpha k(t) / \beta-\epsilon_{0} \\
\beta>\sum_{i} \eta_{i}{ }^{\prime} / \zeta_{i}{ }^{\prime}\left(\theta_{i}{ }^{\prime}-1\right), \quad 1<\theta_{i}{ }^{\prime}<2, \text { for all } i .
\end{gathered}
$$

A combination of Lemmas $1-3$ and Results 1 and 2 yields the following.

\section{Result 3}

When $\phi(\sigma) \subset P_{M}$, the integral (with $\alpha, \beta>0$, and $T$ so chosen that $\sigma(t)$ attains its maximum value at $t=T$ )

$$
\begin{aligned}
I^{a}= & \int_{0}^{T} k(t) \phi(\sigma) \\
& \cdot\left[\alpha \sigma+\beta(d \sigma / d t)+\left(Z_{1}+Z_{2}+Z_{1}^{a}+Z_{2}^{a}\right) \sigma(t)\right] d t
\end{aligned}
$$

is positive (except for a constant term of the form $d_{1}(|\sigma(0)|)$ in $\left.(7)\right)$ if (for some $\epsilon_{0}>0$ )

$$
\begin{aligned}
& -\min _{i}\left(\zeta_{i} \theta_{i},\left(2-\theta_{i}{ }^{\prime}\right) \zeta_{i}{ }^{\prime}\right) k(t) \\
& \quad \leq(d k / d t) \delta_{\max } \\
& \quad \leq \min _{i}\left(\alpha / \beta, \nu_{i} \mu_{i},\left(2-\nu_{i}{ }^{\prime}\right) \mu_{i}{ }^{\prime}\right) k(t)-\epsilon_{0}
\end{aligned}
$$

$\beta>\sum_{i}\left[\eta_{i} /\left(1-\theta_{i}\right) \zeta_{i}\right]+\sum_{i}\left[\eta_{i}{ }^{\prime} /\left(\theta_{i}^{\prime}-1\right) \zeta_{i}^{\prime}\right]$,

$0 \leq \nu_{i}<1, \quad 1<\nu_{i}^{\prime} \leq 2$,

$0<\theta_{i}<1, \quad 1<\theta_{i}{ }^{\prime}<2$, for all $i$.

\section{Lemma 7}

If $\mid f(t): \leq r_{2} \exp \left(-r_{0} t\right)$ with $r_{2}, r_{0}>0$, then there exists a constant $R_{1}{ }^{\prime}$ independent of $T$ such that

$$
\left|\int_{0}^{T^{\prime}} k(t) \phi(\sigma)\left[\left(Z_{1}{ }^{a}+Z_{2}^{a}\right) f(t)\right] d t\right| \leq R_{1} \sup _{0 \leq t \leq T}|\sigma(t)| .
$$

Proof: The proof is similar to that found in Popov [1].

\section{B. Main Results}

Having settled the preliminaries, a major result (Theorem 3) of this section can be stated. This theorem, believed to be new, shows that the price to be paid for the introduction of a noncausal function into the stability multiplier is a lower bound on $d k / d t$. Theorem 3 includes Theorem 1 as a special case. When $\phi(\sigma) \subset P_{M}$, the theorem still holds after casting out inadmissible terms from the multiplier.

\section{Theorem 3}

The system (5) is absolutely stable for $\phi(\sigma) \subset P_{M O}$ if there exist constant $\alpha, \beta>0 ; \gamma_{i}, \gamma_{i}{ }^{\prime}, n_{i}, \eta_{i}{ }^{\prime}, \mu_{i}, \mu_{i}{ }^{\prime} \geq 0 ; \zeta_{i}, \zeta_{i}{ }^{\prime}>$ $0 ; 0 \leq \nu_{i}<1,1<\nu_{i}^{\prime} \leq 2,0<\theta_{i}<1,1<\theta_{i}{ }^{\prime}<2$, for all $i$,

$$
\beta>\sum_{i}\left[\eta_{i} /\left(1-\theta_{i}\right) \zeta_{i}\right]+\sum_{i}\left[\eta_{i}^{\prime} /\left(\theta_{i}^{\prime}-1\right) \zeta_{i}^{\prime}\right]
$$

and a multiplier

$$
z(s)=\alpha+\beta s+z_{1}(s)+z_{2}(s)+z_{1}{ }^{a}(s)+{z_{2}}^{a}(s)
$$

such that

a) $\operatorname{Re} z(j \omega) G(j \omega) \geq 0$, for all real $\omega$, and for some $\epsilon_{0}>0$, as small as desired,

b) $\min _{i}\left(\zeta_{i} \theta_{i},\left(2-\theta_{i}{ }^{\prime}\right) \zeta_{i}{ }^{\prime}\right) k(t)$

$$
\begin{aligned}
& \leq(d k / d t) \delta_{\max } \\
& \leq \min _{i}\left(\alpha / \beta, \nu_{i} \mu_{i},\left(2-\nu_{i}{ }^{\prime}\right) \mu_{i}{ }^{\prime}\right) k(t)-\epsilon_{0} .
\end{aligned}
$$

Proof: It is similar to the proof of Theorem 1. The points of departure are 1) the negative terms due to $Z_{1}{ }^{a}$ and $Z_{2}{ }^{a}$ are to be dominated by the positive contribution of $\beta s$ (see preliminary results);2) Lemma 7 is to be taken into account.

As in the proof of Theorem 1, hypothesis a) leads to the inequality

$$
\begin{aligned}
\int_{0}^{T} k(t) \phi(\sigma)\left[\alpha \sigma+\beta(d \sigma / d t)+\left(Z_{1}+Z_{2}+Z_{1}^{a}+Z_{2}^{a}\right) \sigma(t)\right] d t \\
\leq \int_{0}^{T} k(t) \phi(\sigma)[\alpha f+\beta(d f / d t) \\
\left.\quad+\left(Z_{1}+Z_{2}+Z_{1}^{a}+Z_{2}^{a}\right) f(t)\right] d t \\
\leq R_{1} \sup _{0 \leq t \leq T}|\sigma(t)|
\end{aligned}
$$

the constant $R_{1}$ being independent of $T$. 
Concerning Result 2, let

$$
\sigma_{3}(T)=\xi_{i}^{\prime} \int_{T}^{\infty} \exp \left[\zeta_{i}^{\prime}(T-\tau)\right] \sigma(\tau) d \tau
$$

For the definition of $\sigma_{2}(T)$, see (29). Hypothesis b) on application to the left-hand side of (44) gives, as in the proof of Theorem 1, for an $\epsilon_{2}(t) \geq \epsilon_{0}>0$, for all $t$, and a constant $R_{0}$ independent of $T$,

$$
\begin{aligned}
\int_{0}^{T} \epsilon_{2}(t) \phi(\sigma) \sigma d t+k(T)\left\{\beta \Phi(\sigma(T))-\sum_{i} \eta_{i} \Phi\left(\sigma_{2}(T)\right) / \xi_{i}\right. \\
\left.\quad-\sum_{i} \eta_{i}{ }^{\prime} \Phi\left(\sigma_{3}(T)\right) / \xi_{i}{ }^{\prime}\right\} \\
\quad+\left(\text { nonnegative terms due to } Z_{1}, Z_{2}, Z_{1}{ }^{a}, Z_{i}{ }^{a}\right) \\
\leq R_{0}+R_{1} \sup _{0 \leq t \leq T}|\sigma(t)| .
\end{aligned}
$$

Since the inequality (45) has been stated for every positive $T$, it holds in particular for $T$ so chosen as to permit $\sigma(t)$ to attain its maximum value at $t=T$. Therefore, $\sigma(t)$ in $\Phi(\sigma(T))$ of the left-hand side of (45) can be replaced by $\sigma_{\max }$ without affecting the inequality. This enables one to use Result 3, which guarantees that the expression inside the braces of (45) is positive and greater than or equal to $\beta_{0} k(T) \Phi(\sigma(T))$ for some $\beta_{0}>0$. Consequently,

$\int_{0}^{T} \epsilon_{2}(t) \phi(\sigma) \sigma d t+\beta_{0} k(T) \Phi(\sigma(T))+$ (nonnegative terms)

$$
\leq R_{0}+R_{1} \sup _{0 \leq t \leq T}|\sigma(t)|
$$

from which stability and asymptotic stability of $\sigma(t)$ ensue as in Popov's proof [1].

Corollary: If $\phi(\sigma) \subset P_{M}$, the terms $z_{2}(s)$ and $z_{2}{ }^{a}(s)$ are inadmissible in the multiplier. Hypothesis b) accordingly reads

$-\min _{i} \zeta_{i} \theta_{i} k(t) \leq(d k / d t) \delta_{\max } \leq \min _{i}\left(\alpha / \beta, \nu_{i} \mu_{i}\right) k(t)-\epsilon_{0}$.

\section{Potcer Law Nonlinearity}

The present aim is to include $z_{1}{ }^{a}(s)$ and $z_{2}^{a}(s)$ in the multiplier of Theorem 2. By doing so, Theorem 3 is generalized.

Because the property (2) of $\phi(\sigma)$ is common for both $\phi(\sigma) \subset P_{P}{ }^{m_{0}}$ and $\phi(\sigma) \subset P_{M}$, Result 1 concerning $z_{1}{ }^{a}(s)$ holds for $\phi(\sigma) \subset P_{P} m_{0}$. As regards $z_{2}^{a}(s)$, an analysis similar to the one leading to Result 2 gives the following.

\section{Result 4}

When $\phi(\sigma) \subset P_{P}^{m_{0}}$ and $c \neq 0$, the integral (with $\alpha, \beta>0$ and $T$ chosen so as to make $\sigma(l)$ attain its maximum at $t=T)$

$$
I_{0 p}{ }^{a}=\int_{0}^{T} k(t) \phi(\sigma)\left[\alpha \sigma+\beta(d \sigma / d t)+\left(Z_{:}^{a} \sigma(t)\right)\right] d t
$$

is positive (except for a constant term of the form $d_{1}(|\sigma(0)|)$ in $\left.(7)\right)$ if (for some $\epsilon_{0}>0$ )

$$
\begin{aligned}
-\min _{i}\left(1+c^{2}-c^{2} \theta_{i}{ }^{\prime}\right) \zeta_{i}{ }^{\prime} k(t) & \leq(d k / d t) \delta_{\max } \\
& \leq(\alpha / \beta) k(t)-\epsilon_{0}
\end{aligned}
$$

$\beta>\sum_{i} \eta_{i}{ }^{\prime} / c^{2}\left(\theta_{i}^{\prime}-1\right) \zeta_{i}^{\prime}, \quad 1<\theta_{i}^{\prime}<1+1 / c^{2}, \quad$ for all $i$.

A combination of the Corollary to Lemma 6, Result 1, and Result 4 gives the following.

\section{Result j}

When $\phi(\sigma) \subset P_{P}^{m_{0}}$ and $c \neq 0$, the integral (with $\alpha, \beta>0$ and $T$ chosen so as to allow $\sigma(t)$ to reach its maximum value at $t=T$ )

$$
\begin{aligned}
I_{p}{ }^{a}= & \int_{0}^{T} k(t) \phi(\sigma) \\
& \cdot\left[\alpha \sigma+\beta(d \sigma / d t)+\left(Z_{1}+Z_{2}+Z_{1}^{a}+Z_{2}^{a}\right) \sigma(t)\right] d t
\end{aligned}
$$

is positive (except for a constant term of the form $d_{1}(|\sigma(0)|)$ in (7)) if (for some $\epsilon_{0}>0$ )

$$
\begin{aligned}
& -\min _{i}\left(\theta_{i} \zeta_{i},\left(1+c^{2}-c^{2} \theta_{i}{ }^{\prime}\right) \zeta_{i}\right) k(t) \\
& \leq(d k / d t) \delta_{\max } \\
& \leq \min _{i}\left(\alpha / \beta, \nu_{i} \mu_{i},\left(1+c^{2}-c^{2} \nu_{i}^{\prime}\right) \mu_{i}{ }^{\prime}\right) k(t)-\epsilon_{0} \\
& \beta>\sum_{i} \eta_{i} /\left(1-\theta_{i}\right) \zeta_{i}+\sum_{i} \eta_{i}{ }^{\prime} / c^{2}\left(\theta_{i}{ }^{\prime}-1\right) \zeta_{i}{ }^{\prime}, \\
& 0 \leq \nu_{i}<1, \quad 1<\nu_{i}^{\prime} \leq 1+1 / c^{2}, \\
& 0<\theta_{i}<1, \quad 1<\theta_{i}^{\prime}<1+1 / c^{2}, \text { for all } i .
\end{aligned}
$$

A limiting case of Result 5 is the following.

\section{Result 6}

When $\phi(\sigma)=\sigma$, the integral (with $\alpha, \beta>0$ and $T$ chosen so as to allow $\sigma(t)$ attain its maximum value at $t=T)$

$$
\begin{aligned}
I_{L}= & \int_{0}^{T} k(t) \phi(\sigma) \\
& \cdot\left[\alpha \sigma+\beta(d \sigma / d t)+\left(Z_{1}+Z_{2}+Z_{1}^{a}+Z_{2}^{a}\right) \sigma(t)\right] d t
\end{aligned}
$$

is positive (except for a constant term of the form $d_{1}(|\sigma(0)|)$ in (7)) if (for some $\epsilon_{0}>0$ )

$$
\begin{aligned}
-2 \min _{i}\left(\theta_{i} \zeta_{i}, \zeta_{i}\right) k(t) & \leq d k / d t \\
& \leq 2 \min _{i}\left(\alpha / \beta, \nu_{i} \mu_{i}, \mu_{i}^{\prime}\right) k(t)-\epsilon_{0}
\end{aligned}
$$

$$
\begin{aligned}
& \beta>\sum_{i} \eta_{i} /\left(1-\theta_{i}\right) \zeta_{i}+\sum_{i} \eta_{i}{ }^{\prime}\left(\theta_{i}{ }^{\prime}-1\right) / \zeta_{i}{ }^{\prime}, \\
& \quad 0 \leq \nu_{i}<1, \quad \nu_{i}{ }^{\prime}>1, \quad 0<\theta_{i}<1, \quad \theta_{i}{ }^{\prime}>1, \text { for all } i .
\end{aligned}
$$

Based on the preceding results, the following theorem generalizes Theorem 3. Its proof resembles the proof of Theorem 3 and is hence not given. 


\section{Theorem 4}

The system governed by (5) is absolutely stable for $\phi(\sigma) \subset P_{P} m_{0}, c \neq 0$, if there exist constants $\alpha, \beta>0$; $\eta_{i}, \eta_{i}{ }^{\prime}, \gamma_{i}, \gamma_{i}{ }^{\prime}, \mu_{i}, \mu_{i}{ }^{\prime} \geq 0 ; 0 \leq \nu_{i}<1,1<\nu_{i}{ }^{\prime} \leq\left(1+1 / c^{2}\right)$, $0<\theta_{i}<1,1<\theta_{i}{ }^{\prime}<\left(1+1 / c^{2}\right)$, for all $i$,

$$
\beta>\sum_{i} \eta_{i} /\left(1-\theta_{i}\right) \zeta_{i}+\sum_{i}\left[\eta_{i}{ }^{\prime} / c^{2}\left(\theta_{i}{ }^{\prime}-1\right) \zeta_{i}{ }^{\prime}\right]
$$

and a multiplier

$$
z(s)=\alpha+\beta s+z_{1}(s)+z_{2}(s)+z_{1}{ }^{a}(s)+z_{2}{ }^{a}(s)
$$

such that

a) $\operatorname{Re} z(j \omega) G(j \omega) \geq 0$, for all real $\omega$, and, for some $\epsilon_{0}>0$, as small as desired,

b) $-\min \left(\theta_{i} \zeta_{i},\left(1+c^{2}-c^{2} \theta_{i}{ }^{\prime}\right) \zeta_{i}{ }^{\prime}\right) k(t)$

$$
\begin{aligned}
& \leq(d k / d t) \delta_{\max } \\
& \leq \min _{i}\left(\alpha / \beta, \nu_{i} \mu_{i},\left(1+c^{2}-c^{2} \nu_{i}{ }^{\prime}\right) \mu_{i}{ }^{\prime}\right) k(t)-\epsilon_{0} .
\end{aligned}
$$

Corollary 1: When $e=1$ (or $\phi(\sigma) \subset P$ :oo), Theorem 3 is obtained.

Corollary 2: (Based on Result 6) When $\phi(\sigma)=\sigma$, the linear system governed by (5) is asymptotically stable if there exist constants $\alpha, \beta>0 ; \eta_{i}, \eta_{i}{ }^{\prime}, \gamma_{i}, \gamma_{i}{ }^{\prime}, \mu_{i}, \mu_{i}{ }^{\prime} \geq 0 ; 0 \leq$ $\nu_{i}<1, \nu_{i}^{\prime}>1,0<\theta_{i}<1, \theta_{i}^{\prime}>1$, for all $i$,

$$
\beta>\sum_{i}\left[\eta_{i} /\left(1-\theta_{i}\right) \zeta_{i}\right]+\sum_{i}\left[\eta_{i}{ }^{\prime}\left(\theta_{i}^{\prime}-1\right) / \zeta_{i}{ }^{\prime}\right]
$$

and a multiplier

$$
z(s)=\alpha+\beta s+z_{1}(s)+z_{2}(s)+z_{1}{ }^{a}(s)+z_{2}{ }^{a}(s)
$$

such that

a) $\operatorname{Re} z(j \omega) G(j \omega) \geq 0$ for all real $\omega$, and, for some $\epsilon_{0}>0$, as small as desired,

b) $-2 \min _{i}\left(\theta_{i} \zeta_{i}, \zeta_{i}{ }^{\prime}\right) k(t) \leq d k / d t$

$$
\leq 2 \min _{i}\left(\alpha / \beta, \nu_{i} \mu_{i}, \mu_{i}{ }^{\prime}\right) k(t)-\epsilon_{0}
$$

\section{Remarks}

1) The present method of introducing a noncausal function into the stability multiplier is different from O'Shea's [7] correlation method developed explicitly for a time-invariant system. (See Zames and Falb [9] for a rigorous treatment of the correlation method).

2) The multiplier introduced by O'Shea [7] is very general, but the accompanying time domain restriction on the multiplier is not always easy to verify. The stability multiplier of the paper contains an RC-RL reflected impedance, and there is no explicit time domain restriction on it. It is possible to extend the class of multipliers (for $\phi(\sigma)\left(P_{M O}, P_{P}{ }^{m o}\right)$ by considering biquadratic functions.

3) It has been found that the correlation technique, on extension to the time-varying feedback problem, leads to an inconsequential bound on $d k / d t$; namely, $k(t)$ is either a constant or a nonincreasing function of time. However, if the time-varying gain $k(t)$ is periodic, one can arrive at useful conditions for absolute stability of the system in terms of a special multiplier; a detailed correlationbound analysis is not essential. This contribution will appear elsewhere.

\section{E. Examples}

1) Let

$$
G(s)=s /(s+1)^{3}, \quad \phi(\sigma) \subset P_{M} .
$$

With constant linear feedback, the system is asymptotically stable for all gains $\subset[0, \infty)$. Choose

$$
\begin{aligned}
z(s) & =2+s+[1-2 /(6-s)] \\
& =2+s+(4-s) /(6-s) .
\end{aligned}
$$

$\xi=2, \zeta=6, \alpha=2, \beta=1$. Note that $\zeta>\xi, \beta>1 / \xi$. Also $0<\delta_{\max } \leq 1$. Corollary 1 of Theorem 3 is applicable. It can be verified that $\operatorname{Re} z(j \omega) G(j \omega) \geq 0$, for all real $\omega$. The system is, therefore, absolutely stable for $\phi(\sigma) \subset P_{M}$ if, for some $\epsilon_{0}>0$, as small as desired

$$
-4 k(t) \leq(d k / d t) \delta_{\max } \leq 2 k(t)-\epsilon_{0} .
$$

When the system is linear, $\delta_{\max }=1 / 2$ and the preceding bound on $d k / d t$ becomes

$$
-8 k(t) \leq d k / d t \leq 4 k(t)-\epsilon_{0} .
$$

Observe that for the linear system, using positive real multipliers alone, it is unlikely that a result better than $d k / d t \leq 2 k(t)-\epsilon_{0}$ (for asymptotic stability) can be established.

2) Let

$G(s)=s^{3} /\left(s^{5}+5 s^{4}+4 s^{3}+3 s^{2}+2 s+5\right), \quad \phi(\sigma) \subset P^{3}$.

With linear constant feedback, the system is asymptotically stable for all gains $C[0, \infty)$. Choose

$$
z(s)=2 s+4+[1+1 /(6-s)] .
$$

Here $\xi^{\prime}=1, \zeta^{\prime}=6, \alpha=4, \beta=2, \zeta^{\prime}-\xi^{\prime}>0, \beta>1 / \xi^{\prime}$, $0<\delta_{\max } \leq 1$. Theorem 3 is applicable. It is easy to verify that $\operatorname{Re} 2(j \omega) G(j \omega) \geq 0$, for all real $\omega$.

Therefore, the nonlinear time-varying feedback system is absolutely stable for $\phi(\sigma) \subset P_{\text {MO }}$ if, for some $\epsilon_{0}>0$, as small as desired,

$$
-5 k(t) \leq(d k / d t) \delta_{\max } \leq 2 k(t)-\epsilon_{0}
$$

When the system is linear, $\delta_{\max }=1 / 2$. The preceding constraint on $d k / d t$ becomes

$$
-10 k(t) \leq d k / d t \leq 4 k(t)-\epsilon_{0} .
$$

Observe that in the linear time-varying case, because of the third-order zero in $G(s)$, it is not possible to find a positive real $z(s)$ such that $z(s) G(s)$ is strictly positive real. Consequently Gruber and Willems' [8] criterion for the linear time-varying system is not applicable here. 


\section{Conclusions}

New absolute stability criteria are derived for a nonlinear time-varying feedback system illustrated in Fig. 1. The classes of the nonlinearities considered are $P_{\mathrm{H}}, P_{\mathrm{MO}}$, and $P_{P} m_{0}$ (with $m_{0} \geq 1$ ). The criteria are expressed in terms of a multiplier containing causal and noncausal functions. A significant outcome of the presence of noncausal functions in the multiplier is that absolute stability oan be established in cases where a purely causal multiplier is ineffective because the phase angle of $G(s)$ is outside the $\pm 90^{\circ}$ band in many intervals along the $j \omega$ axis; in return, $d k / d t$ is to be bounded from below also. Such a lower bound on $d k / d t$ appears to be the first of its lind. As in earlier investigations, the causal part of the multiplier gives rise to an upper bound on $d k / d t$. These bounds on $d k / d t$ are dependent on the form of the nonlinearity and the multiplier chosen.

The search for a $z(s)$ to satisfy hypothesis a) of the theorems is rather cumbersome. It would be very useful in practice to have a direct method by which a candidate for $z(s)$ is obtained directly from the phase angle characteristic of $G(s)$. This is an area deserving study.

\section{ACKNOWLEDGMENT}

The author is grateful to Prof. H. X. Ramachandra Rao for his interest and stimulation, and to Dr. M. A. L. Thathachar for useful discussions and advice. Thanks are extended to the reviewers whose suggestions have improved the value of this paper.

\section{REFERENCES}

[1] Y. M. Popoy, "Absolute stability of nonlinear systems of automatic control," Auto. and Remote Control, pp. 857-875, IIarch 1962.

[2] G. Zames, "On the stability of nonlinear time varying feedback sistems," Proc. 1964 NEC, vol. 20, pp. 725-730.
"Nonlinear time varying feedback systems-conditions for $L_{\infty}$-boundedness derived using conje operators on exponentjally weighted spaces," Proc. Brd Ann. Allerton Conf. Circuit and System Theory, 1965, pp. 460-472.

[3] Y. S. Cho and K. S. Narendra, "Stability of nonlinear time varying feedback systems," Automatica, vol. 4 , pp. 309-31i, 1968.

[4] K. S. Narendra and J. H. Taylor, "Lyapunov functions for nonlinear time varying feedback systems," Inform. and Control, vol. 13, pp. 378-387, 1968.

[j] K. S. Narendra and C. P. Neuman, "Stability of a class of differential equations with single monotone nonlinearity," SIA II J. Control, vol. 4, pp. 295-308, 1966.

[6] R. W. Brockett and J. L. Willems, "Frequency domain stability criteria, pt. I," IEEE Trans. Automatic Control, vol. AC-10, pp. 255-261, JuJy 1965.

-. "Frequency domain stability criteria, pt. II," IEEE Trans. Automatic Control, vol, AC-10, pp. 407-413, December 1965 .

[7] R. P. O'Shea, "An improved frequency time domain stability cliterion for autonomous continuous systems, " IEEE Trans. Automatic Control, vol. AC-12, pp. 725-731, December 1967.

[8] M. Gruber and J. L. Willems, "On a generalization of the circle criterion," Proc. 4th Ann. Allerton Conf. Circuit and System Theory, 1966, pp. 827-848.

[9] G. Zames and P. I. Falb, "Stability conditions for systems with monotone and slope-restricted nonlinearities," SIA $M J$. Control, vol. 6, no. 1, pp. 89-108, 1968.

[10] M. A. L. Thathachar, M. D. Srinath, and H. K. Ramapriyan, "On a modified Lur'e problem," IEEE Trane. Automatic Control, vol. AC-12, pp. $731-740$, December 1967 .

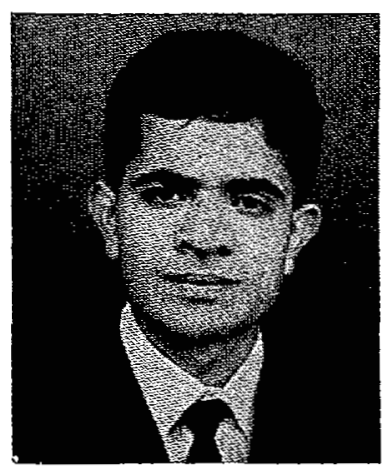

Yedatore V. Venkatesh was borm in Mysore, South India. He was educated at the National Institute of Engineering, Mysore, and received the M.E. and Ph.D. degrees in electrical engineering from the Indian Institute of Science, Bangalore, in 1965 and 1970 , respectively.

He is presently with the Department of Electrical Engineering at the Indian Institute of Science. His areas of interest include optimal control and automata theory. 